\title{
The role of suppressor protein in acquired resistance to viral infection
}

Tleukulova Zh.*, Amanbayeva U., Akbassova A., Zhangazin S., Iksat N., Dildabek A., Ilyasova B., Stamgaliyeva Z., Masalimov Zh., Omarov R.

L.N. Gumilyov Eurasian National University, Nur-Sultan, Kazakhstan

*e-mail: zhanerke.birzhan@gmail.com

Plants are constantly exposed to abiotic and biotic stresses. One of the main types of biotic stress is viral agents. The viruses cause tremendous damage; therefore, plant viral diseases are an important problem in phytopathology, as they cause great damage to agriculture. We have been studying TBSV, which has a broad host range under experimental conditions and has been reported to infect over 120 plant species spanning 20 families. Tomato bushy stunt virus (TBSV) is the type species of the Tombusvirus family. TBSV has a single +ssRNA genome with length of about $4800 \mathrm{nt}$, which is wrapped by 180 subunits of capsid protein. The virus contain 5 open reading frames: p33 and p92 responsible for replicase, which translated from genomic RNA, capsid protein p41 is translated from subgenomic RNA1, whereas $\mathrm{p} 22$ and $\mathrm{p} 19$ from subgenomic RNA2. The $\mathrm{p} 22$ protein is a movement protein that is required for the virus to spread from cell to cell. P19 is a major viral pathogenicity determinant and functions as a suppressor of defensive mechanism RNAi. This protein binds siRNAs and prevent their incorporation into RISC, thereby allowing viral propagation in the host. Therefore, manipulations with this protein can impart resistance to viral pathogen. Nicotiana benthamiana plants, which initially inoculated with mutant of TBSV-157 survived, due to absence p19, then these plants were grown. After that, the seeds from these plants $(157 \mathrm{~s} \mathrm{N.b.)}$ were collected. Collected seeds were planted, and after 30 days growth plants were inoculated with wild type TBSV. For experiments were taken N. benthamina, 157 s N.b. plants, which were infected by TBSV and showed the same symptoms. But only $157 \mathrm{~s} \mathrm{~N}$. benthamiana plants survived after 14 days post inoculation. Taken together, it is possible to assume that the infection with suppressor mutant, may effectively trigger systemic resistance of plants in the next generation. 